\title{
Numerical simulation of a scramjet isolator with thermodynamic nonequilibrium
}

\author{
Romain Fiévet* ${ }^{*}$ Heeseok $\mathrm{Koo}^{\dagger}$ and Venkat Raman ${ }^{\ddagger}$ \\ Department of Aerospace Engineering, The University of Michigan, Ann Arbor, MI 48109-2102, USA
}

\begin{abstract}
Flow inside the isolator of a scramjet engine is likely to be in thermal non-equilibrium due to successive shock-based compressions and expansions. Given the short flow-through timescales in such engines, the flow might not reach equilibrium even in the fuel injection region. Since the distribution of energy in the internal modes affects chemical reactions, non-equilibrium has been shown to have significant impact on ignition and flame stabilization. In this study, detailed numerical simulation of a supersonic turbulent channel flow coupled with a multi-temperature model is used to quantify the impact of thermodynamic non-equilibrium on the shock train structure inside the isolator and the flow characteristics at the outlet.
\end{abstract}

\section{Introduction}

Scramjet engines rely on shock-generated compression to maintain a stable combustion zone in the flow path. In general, the inlet of the engine is separated from the combustor through a section called the isolator, that maintains the shock structures that provide the compression. The length of the isolator is conservatively determined so that changes in flow conditions (over a flight path) do not lead to loss of compression through inlet unstart, a process by which oblique shock structures in the isolator are displaced upstream leading to a detached bow shock at the entrance of the engine. Chief among disturbances that lead to unstart is the performance of the combustion region, more particularly, the heat addition in the reaction region of the engine. Hence, any flow feature that affects the combustion process could potentially undermine the stability of the scramjet engine.

In this regard, thermal nonequilibrium could play an important role. It is well known that all the internal motions of the molecules may not relax to their equilibrium conditions rapidly following a compression shock. In flow conditions similar to that experienced in scramjet engines, the translational component of the molecular motions will rapidly relax through collisions, leading to a Maxwellian distribution for the molecular velocity. On the other hand, the rotational and vibrational components relax much more slowly, leading to a persistent region of thermal nonequilibrium. This could impact the reacting flow in two different ways. First, for a given total energy, the presence of nonequilibrium implies that more energy is stored in translational mode leading to increased viscosity and higher viscous dissipation. Second, the change in the relative proportion of energy stored in the different modes changes the reaction rates. In fact, a prior study from our research group ${ }^{1}$ has shown that such nonequilibrium processes could significantly alter the location of the flame stabilization region. Ongoing studies show that the effect on chemical reactions is non-trivial and depend on the reactants as well as the nature of the chemical reactions. Consequently, understanding the generation of such nonequilibrium is important for the design of reliable scramjet engines.

A defining feature of the inlet-isolator flow is the shock-turbulence interaction. In such flows, the coupling between thermodynamic non-equilibrium and turbulence is highly complex due to the large variations in pressure and temperature across the flow cross-section. A thorough description of the flow would require the computation of each specie's translational, rotational and vibrational state energetic distribution, which

\footnotetext{
*PhD Student, AIAA Student Member.

${ }^{\dagger}$ Post-Doctoral Research Fellow, AIAA Member.

¥Associate Professor, AIAA Senior Member,

Copyright (C) 2015 by Romain Fiévet. Published by the American Institute of Aeronautics and Astronautics, Inc. with
} permission. 
would be computationally impracticable. An alternative is to assume that the different modes achieve equilibrium independently in a relatively short time (few collisions) but produce distributions with different temperatures. Since rotational equilibrium is achieved within tens of collisions as previously mentioned, it is essentially in equilibrium with the translational component. In this situation, two temperatures (one each for vibration and translation) is sufficient to describe the population. The two-temperature approach has been used widely in the area of re-entry vehicles and external hypersonics. ${ }^{2,3,4,5}$ In this study, the two-temperature model is used to account for vibrational non-equilibrium and investigate its effect on the thermodynamic state at the end of the isolator.

With this background, the focus of this work is to determine the evolution of vibrational nonequilibrium inside an inlet-isolator section of a scramjet engine. In particular, under fully supersonic flow conditions, the importance of the nonequilibrium processes in different parts of the isolator cross-section is analyzed.

\section{Formulation of the two-temperature model}

To describe the effect of vibrational nonequilibrium on the flow, a two-temperature model that has previously been successfully used in a reacting jet-coflow configuration is implemented. ${ }^{1}$ It requires an additional transport equation for the vibrational energy computed at a certain vibrational temperature. The vibrational energy of each species is computed using

$$
e_{v \alpha}=\int_{T_{\text {ref } \alpha}}^{T_{v}} C_{v \alpha}\left(T^{\prime}\right) d T^{\prime}-\beta_{\alpha} R\left(T_{v}-T_{\text {ref }}\right) .
$$

In the above expression, $C_{v \alpha}$ is the specific heat at constant volume for species $\alpha$, defined relative to a reference temperature $T_{r e f_{\alpha}}$, defined such that there is no vibrational excitation of species $\alpha$ at $T_{r e f_{\alpha}}$. $\beta_{\alpha}$ is $5 / 2$ for diatomic or linear molecules. Here, it has been assumed that the rotational temperature (which is equal to translational temperature, $T_{t}$ ) is always high enough that the rotational levels are fully developped. The above formulation of vibrational energy permits the recovering of the exact energy under thermal equilibrium conditions when $T_{v}=T_{t}$. The vibrational energy of the fluid mixture is given by

$$
E_{v}=\sum_{\alpha=1}^{N} \phi_{\alpha} e_{v \alpha},
$$

where $\phi_{\alpha}$ is the mass fraction of species $\alpha$ in a system of $N$ species. We will consider exclusively non-reacting air composed of oxygen and nitrogen, and operate at temperaure where dissociation can be neglected. The governing equations for fluid flow are then given by

$$
\begin{gathered}
\frac{\partial \rho}{\partial t}+\frac{\partial \rho u_{j}}{\partial x_{j}}=0 \\
\frac{\partial \rho u_{i}}{\partial t}+\frac{\partial \rho u_{j} u_{i}}{\partial x_{j}}=-\frac{\partial P}{\partial x_{i}}+\frac{\partial \tau_{i j}}{\partial x_{j}},
\end{gathered}
$$

where $P$ is the pressure, and $\tau_{i j}$ is the stress tensor that is closed using Newtonian fluid relations.

The energy of the system is divided into two parts: the translational+rotational component and the vibrational component. The transport equations for the translational+rotational energy $\left(E_{t}\right)$ and vibrational energy $\left(E_{v}\right)$ are given by:

$$
\frac{\partial E_{t}}{\partial t}+\frac{\partial u_{j}\left(E_{t}+P\right)}{\partial x_{j}}=\frac{\partial}{\partial x_{j}} k \frac{\partial T_{t}}{\partial x_{j}}-\frac{\partial}{\partial x_{j}}\left(\tau_{i j} u_{i}\right)-Q_{T-V}
$$

where $Q_{T-V}$ denotes the energy exchange between the translational and vibrational modes that leads to full thermal equilibrium.

$$
\frac{\partial \rho E_{v}}{\partial t}+\frac{\partial \rho u_{j} E_{v}}{\partial x_{j}}=\frac{\partial}{\partial x_{j}} k_{v} \frac{\partial T_{v}}{\partial x_{j}}+Q_{T-V} .
$$

The vibrational-translational energy exchange is modeled using a linear relaxation rate with a characteristic time scale $\tau_{v \alpha}: 6,7$

$$
Q_{T-V}=\sum_{\alpha=1}^{N} \rho_{\alpha} \frac{e_{v \alpha}\left(T_{t}\right)-e_{v \alpha}\left(T_{v}\right)}{\tau_{v \alpha}}
$$


The time-scale is found using a mixture rule and the relaxation time scales of pairwise collisions between species in the system:

$$
\tau_{v \alpha}=\frac{1}{\sum_{r=1}^{N} \frac{\chi_{r}}{\tau_{\alpha r}}}
$$

where $\chi_{r}$ is the mole fraction of species $r$. The pairwise relaxation timescale is obtained based on an empirical fit to experimental data ${ }^{6}$

$$
\tau_{\alpha r}=\frac{1}{P} \exp \left[1.16 \times 10^{-3} \mu_{\alpha r}^{\frac{1}{2}} \theta_{v \alpha}^{\frac{4}{3}}\left(T_{t}^{-\frac{1}{3}}-0.015 \mu_{\alpha r}^{\frac{1}{4}}\right)-18.42\right],
$$

where $\mu_{\alpha r}[\mathrm{amu}]$ is the reduced mass of the pair and $\theta_{v \alpha}[\mathrm{K}]$ is the characteristic vibrational temperature of species. The empirical constants are specified to give $\tau_{\alpha r}[\mathrm{~s}]$ for $P[\mathrm{~atm}]$ and $T_{t}[\mathrm{~K}]$.

\section{Flow configuration and simulation conditions}

A direct numerical simulation coupled with a two-temperature model accounting for vibrational nonequilibrium is performed using a geometry similar to the HyShot experiment, ${ }^{8}$ with the primary shock created by a ramp with an incident angle of $18^{\circ}$. The computational domain includes the second oblique shock and is illustrated by the dotted rectangle in Fig. 1. It consists of a $20 \mathrm{~cm}$ long ramp and isolator, and is periodic in the spanwise direction. The isolator is $1 \mathrm{~cm}$ high and $2 \mathrm{~cm}$ wide. The ramp's length is $5 \mathrm{~cm}$ and the isolator is $15 \mathrm{~cm}$ long, and is built such that the reflected oblique shock will prevent an expansion fan to be appear at the inlet of the isolator.

The free stream flow conditions (variables denoted by subscript "0") are set to a constant flight Mach number $M_{0}=8$ with a standard temperature of $T_{0}=300 \mathrm{~K}$, while two atmospheric conditions are investigated. The first set of runs A-C corresponds to a standard sea-level condition : $\rho_{0}=1.18 \mathrm{~kg} \mathrm{~m}^{-3}$, while case D-F corresponds to a low-density high-altitude flight at $30 \mathrm{~km}$, with $\rho_{0}=0.018 \mathrm{~kg} \mathrm{~m}^{-3}$. These two sets of cases provide a large variation in the vibrational relaxation timescale, which is inversely proportional to thermodynamic pressure. We first run a regular simulation that would not take into account vibrational non-equilibrium (cases A and D) before using the relaxation model (cases B and E). Finally, we investigate the effect water vapor might have in the relaxation process by adding an arbitrary amount of $2 \%$ mass fraction of water vapor in cases $\mathrm{C}$ and $\mathrm{F}$ corresponding to a standard ground level value. These six cases are summarized in Table 1, where conditions at the inlet of the computational domain (zone 1) are computed from the free stream conditions using standard oblique shock relations.

The inflow to the geometry is assumed be at a vibrational temperature of $T_{v i b}=300 \mathrm{~K}$, consistent with post-shock conditions after the first oblique shock. The free-stream Reynolds numbers based on the isolator height is $R e_{A}=130000$ for case A. Here, the kinematic viscosity has been increased by a factor of 10 to ensure that the computational grid resolves the near-wall structures in the flow. Similarly, the Reynolds number for the rarified case is $R e_{B}=28000$. The same grid of $\left(n_{x}, n_{y}, n_{z}\right)=(1024,256,128)$ nodes clustered in the wall-direction is used at both conditions, leading to a spatial resolution in wall-unit of $(\Delta x+=80$, $\Delta y+=0.8-12, \Delta z+=30$ ) by taking a representative viscous length scale of $5 \mu \mathrm{m}$. Figure 2 compares the local y-normal grid size to the local viscous length scale for both cases, showing that most of the domain is reasonably well resolved.

Table 1. Summary of different simulations.

\begin{tabular}{cccccccc}
\hline \hline Case & Model & $Y_{H 20}$ & $\rho_{1},\left[{\left.\mathrm{~kg} \cdot \mathrm{m}^{-3}\right]} T_{1},[\mathrm{~K}]\right.$ & $T_{v i b, 1},[\mathrm{~K}]$ & $M_{1}$ & $\mu_{1},\left[\mathrm{~g} \cdot \mathrm{m}^{-1} \cdot \mathrm{s}^{-1}\right]$ \\
\hline $\mathrm{A}$ & Idealgas & 0 & 4.828 & 909 & 300 & 4.2 & 2.50 \\
$\mathrm{~B}$ & Nonequilibrium & 0 & 4.828 & 909 & 300 & 4.2 & 2.50 \\
$\mathrm{C}$ & Nonequilibrium & 0.02 & 4.828 & 909 & 300 & 4.2 & 2.50 \\
$\mathrm{D}$ & Idealgas & 0 & 0.0738 & 909 & 300 & 4.2 & 0.038 \\
$\mathrm{E}$ & Nonequilibrium & 0 & 0.0738 & 909 & 300 & 4.2 & 0.038 \\
$\mathrm{~F}$ & Nonequilibrium & 0.02 & 0.0738 & 909 & 300 & 4.2 & 0.038 \\
\hline \hline
\end{tabular}

The DNS calculation was performed using the compressible flow solver UTComp. ${ }^{9,10,11}$ The solver uses a fifth-order WENO scheme ${ }^{12}$ to compute the nonlinear terms in the momentum and $E_{t}$ equations as well as for scalar flux. A fourth-order central scheme is used for the viscous and diffusion terms. Further details on 
the flow solver are provided in ${ }^{13,14}$. Each simulation was carried out on 1024 processors using MPI-based parallelization for 10 hours. The simulations were initialized from a quiescent flow and continued until all transients were removed from the computational domain. The statistics presented in this preliminary study were collected over one flow-through time after reaching statistical stationarity.

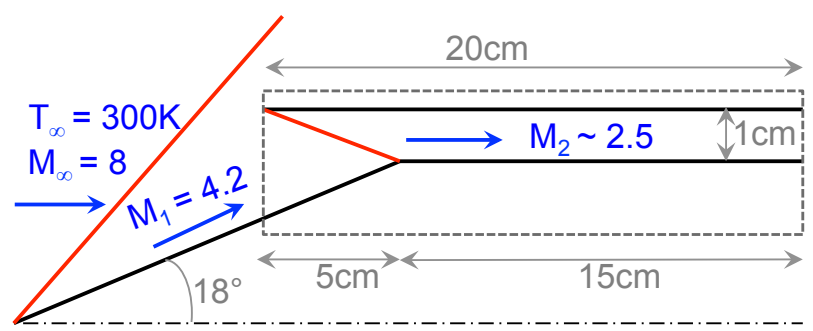

Figure 1. View of the geometry of the HyShot experiment. The dotted box illustrates the present computation domain.
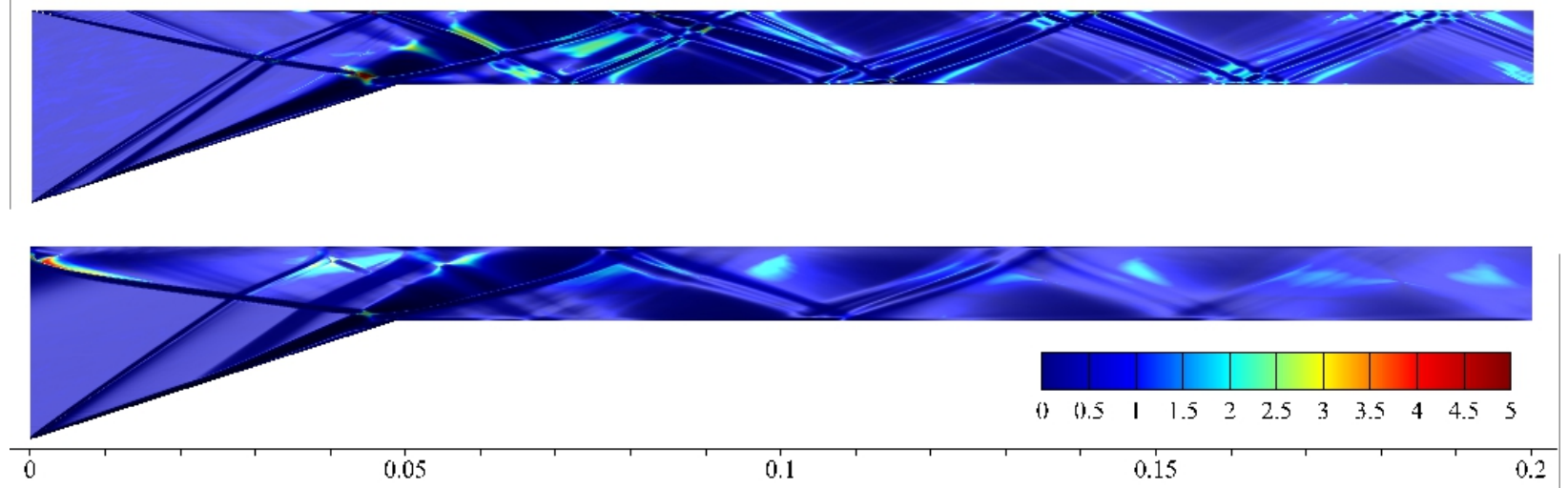

Figure 2. Ratio between the local y-normal grid size to the local viscous length scale for case A (top) and case B (bottom).

\section{Results and discussion}

Figure 3 presents some snapshots of the Mach number, temperature $T$ and density gradient contours presenting the shock structure for equilibrium case A. The ramp angles have been chosen such that oblique shock formed on the upper wall at the inlet does not create an appreciable expansion wave. This contributes to a temperature rise of up to $1800 \mathrm{~K}$ near the centerline, while retaining the supersonic flow structure. It was found that all cases show very similar shock structures, indicating that the nonequilibrium phenomenon does not extensively alter the structure of the supersonic flow.

Figure 4 shows the corresponding time-averaged and spanwise averaged contours for the same case as above. It is seen that the isolator flow exhibits complex shock structures, but the location of the shocks is nearly unaltered from the instantaneous image. This indicates that shock translation is not important in this flow, and that the spanwise variations are also not critical. Hence, the flow is nominally two-dimensional with only the turbulence phenomenon exhibiting the normal three-dimensional structures. The presence of these shock structures will introduce nonequilibrium, which is discussed next.

To quantify the level of nonequilibrium, we define two different indicators. The first is the energy content in the vibrational modes normalized by the equilibrium energy content. This is defined as

$$
\xi=\frac{e_{v i b}}{e_{v i b}^{e q}}
$$

where the superscript eq refers to equilibrium conditions. A second indicator is the normalized difference in the vibrational and translational temperatures, defined as follows:

$$
\theta=\frac{\left|T-T_{v i b}\right|}{T}
$$



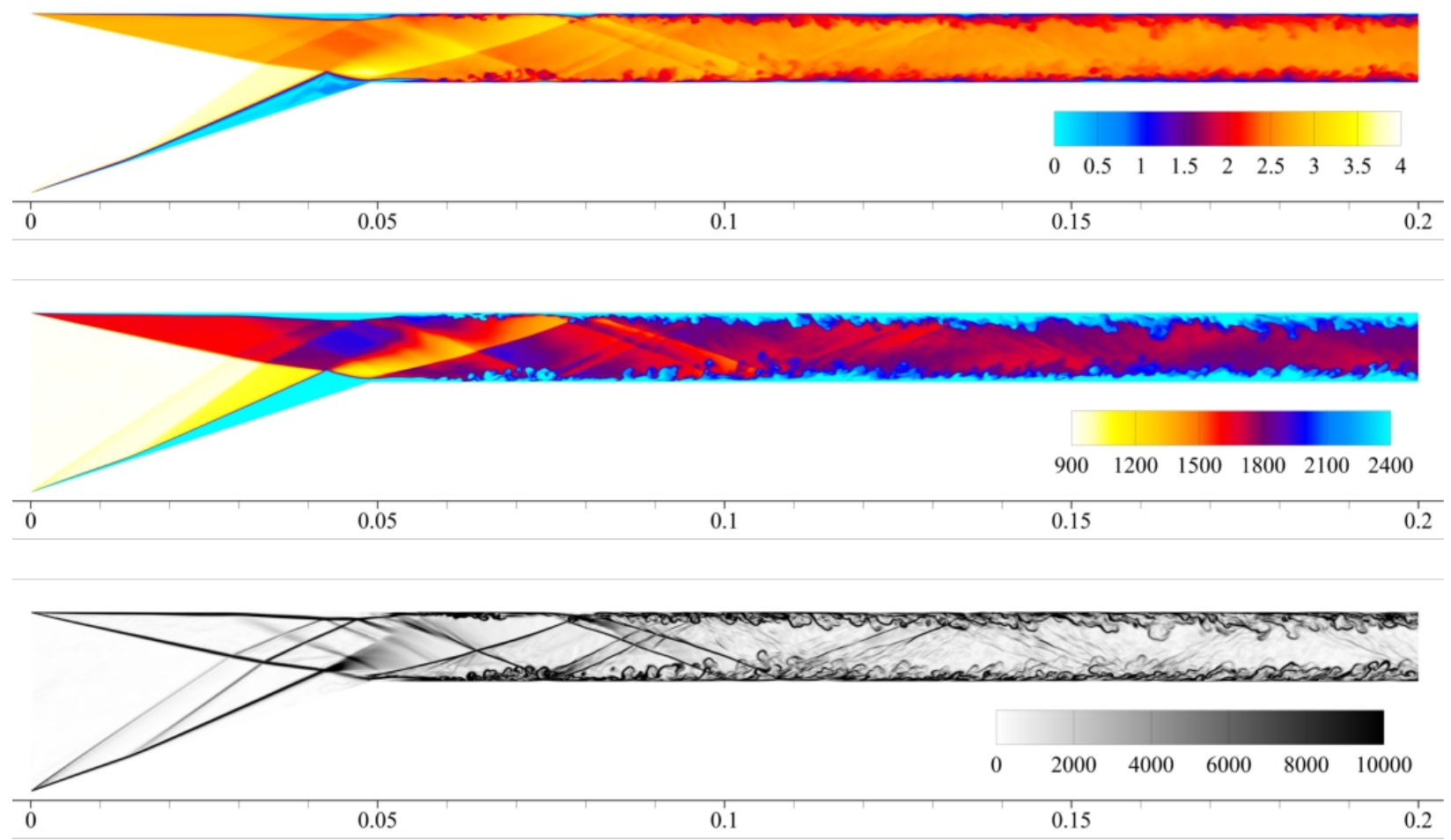

Figure 3. Instantaneous Mach number (top), temperature T (middle) and density gradient (bottom) contours for case A with shadowgraph effects.
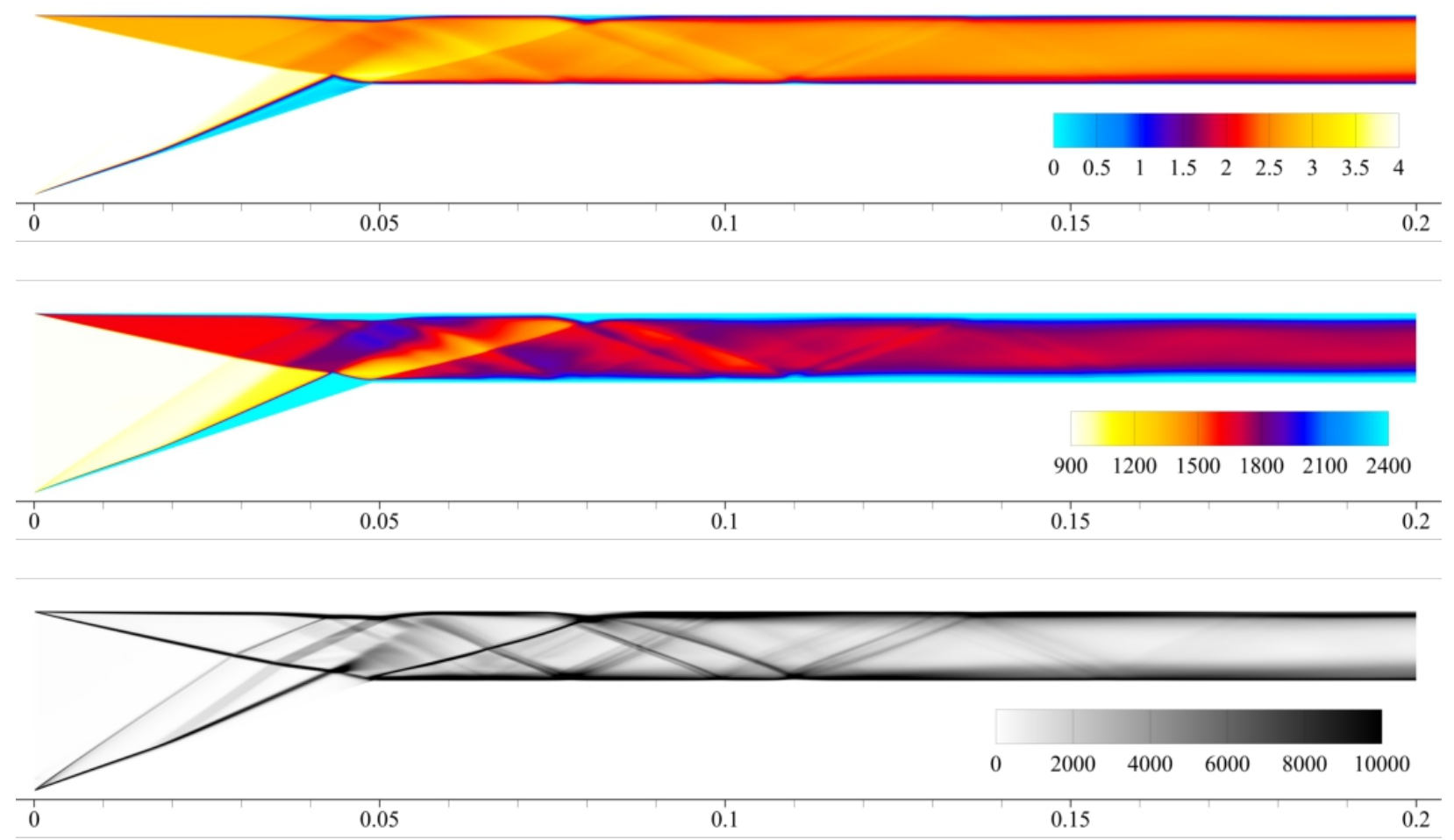

Figure 4. Averaged Mach number (top), temperature T (middle) and density gradients (bottom) contours for case A with shadowgraph effects. 
where $T$ is the translational (also rotational) temperature. $\xi$ shows the fraction of energy stored in the vibrational mode compared to the equilibrium gas, which directly affects the reaction rates. Note that, at equilibrium and at fully excited state (sufficiently high temperatures), the fraction of total energy stored in the vibrational mode will be close to 0.285 for air. $\theta$, on the other hand, provide a more direct measure since the difference in the temperatures determines the rate of relaxation (apart from the thermodynamic properties of the medium).
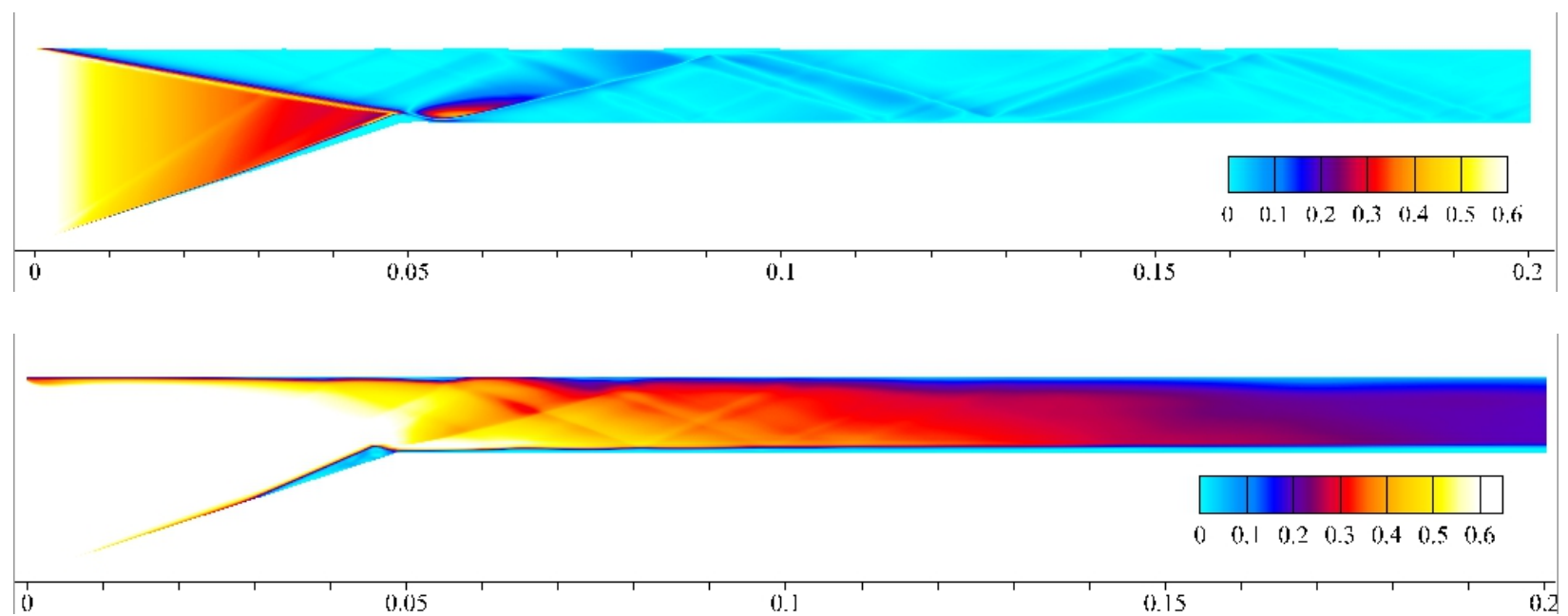

Figure 5. Averaged $\theta$ contours for cases B and E.

Figure 5 shows the averaged contour of $\theta$ for case B and E. The flow is initially in thermal non-equilibrium due to the first oblique shock that is not included in the simulation. Since case A uses low altitude conditions, the pressure post-shock is very high, which is indicative of fast relaxation. This is reflected in the plots where the relaxation is almost complete before reaching the oblique shock in the simulation. Since the pressure rises even further after the first shock, the relaxation is very rapid, with nonequilibrium regions present very close to the shock structures. On the other hand, the low pressure inflow (case E) mimicking the high altitude flight exhibits significant nonequilibrium due to the lower pressure/density. However, in the nearwall regions where the flow velocities are small and the residence time inside the isolator is large, relaxation is able to complete leading to near equilibrium conditions. This variation with cross-section is important to note, since fuel injection strategies further downstream in the combustor could leverage the spatial variations in the degree of nonequilibrium.
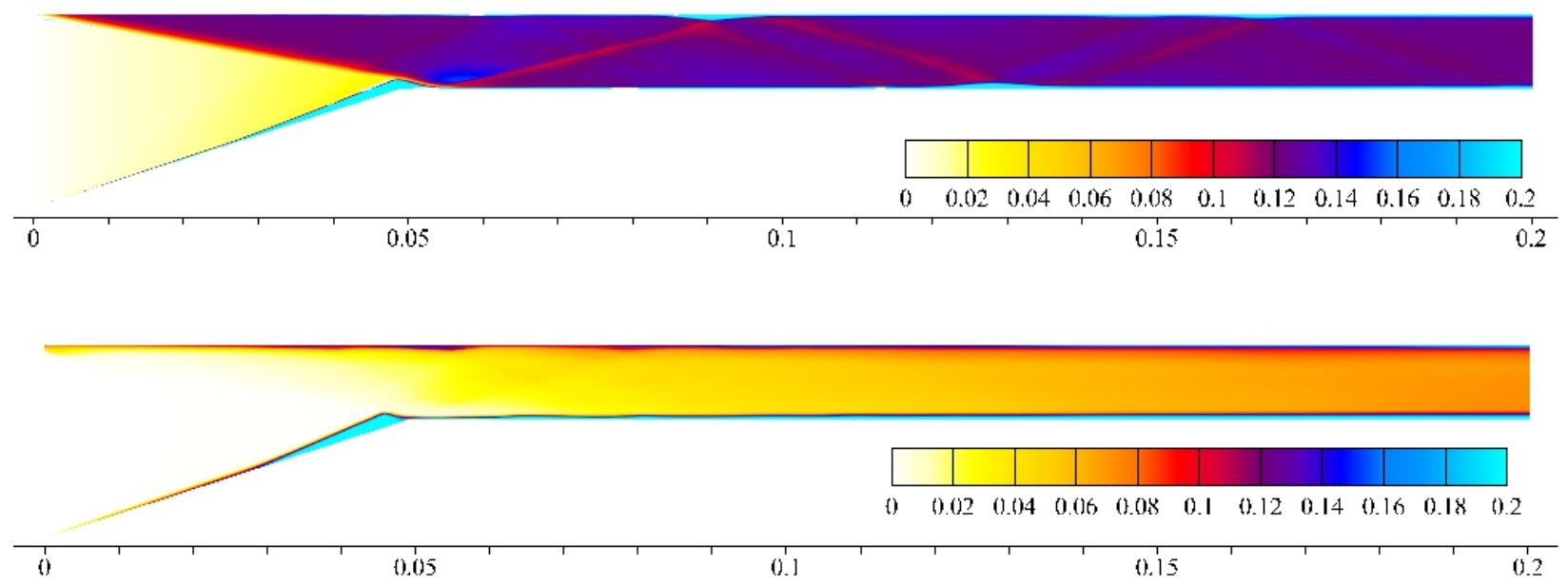

Figure 6. Averaged $\xi$ contours for cases B and E.

The averaged contour of $\xi$ shown in Fig. 6 exhibits similar features, as case B is prompted to reach higher 

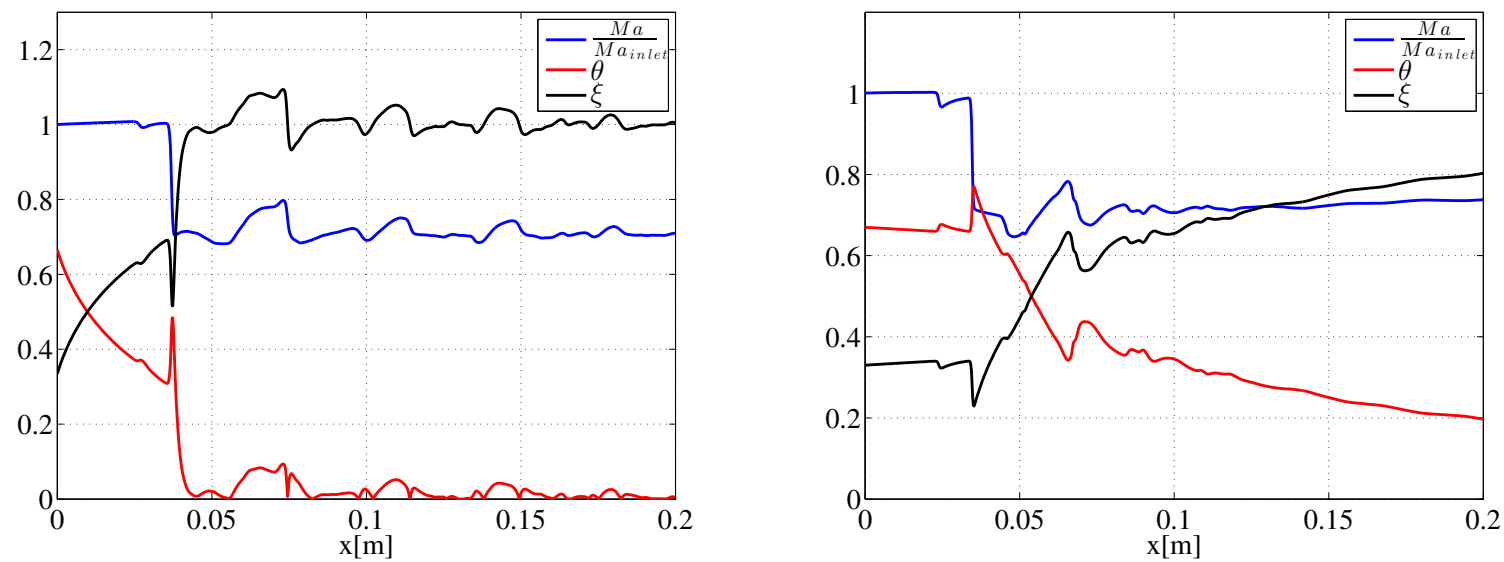

Figure 7. Axial profiles of terms of interests along the isolator centerline for case B (left) and case E (right).

ratio values than case $\mathrm{E}$ due to faster relaxation. Again, the maximum values are found in the slow region of the flow. But there is something else that we can observe when looking the $\xi$ contours : the vibrational modes of the flow in the region between the first and second shock are essentially not excited at all, with less than a percent of energy being stored in those modes. The crossing of the second oblique shock does trigger their excitation (at different rate) as well as the relaxation process as the flow reaches a temperature high enough to unlock these modes. This feature is easily seen in Fig. $7:$ while we observe a strong decay of $\theta$ from the inlet for case B, $\theta$ and $\xi$ start to decay and rise respectively only once they cross the shock at approximately $x=4 \mathrm{~cm}$ for case E.

Now, we can investigate the effect humidity has on the relaxation process by looking at these indicators for cases $\mathrm{C}$ and $\mathrm{F}$. The profiles are shown in Fig. 8 for $\theta$ and Fig. 9 for $\xi$, a ratio of 1 corresponding to a state of equilibrium. The effect of the water vapor on the relaxation process is an overall faster accommodation to local equilibrium, more noticeably for the low-pressure cases $\mathrm{E}$ and $\mathrm{F}$ where the flow hasn't yet reached thermodynamic equilibrium by the end of the isolator. On the other hand, the sea-level flight cases B and $\mathrm{C}$ reach equilibrium by the end of the first half of the domain, due to the much higher ambient pressure inversely proportional to the vibrational relaxation timescale.

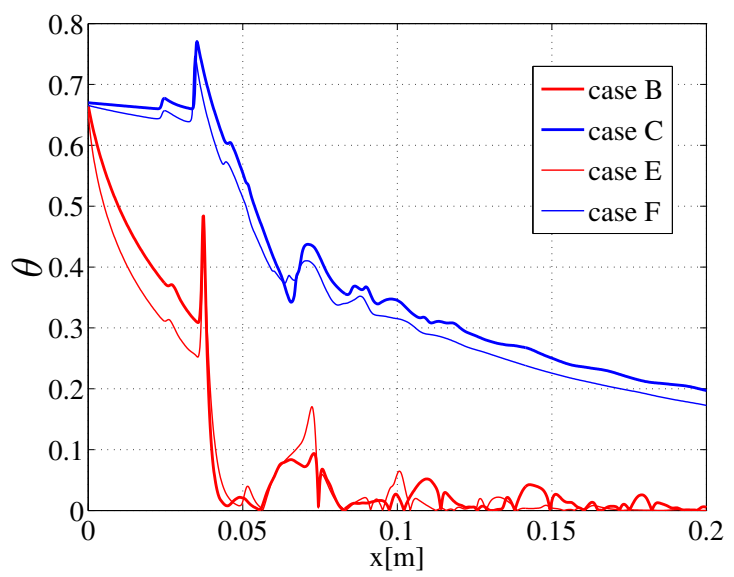

Figure 8. $\theta$ centerline profiles.

As stated previously, our analysis aims at eventually quantify the impact of vibrational non equilibrium on the flow that reaches the combustor, and from a kinetic chemistry point of view we are particularly interested about the influence on the ambient temperature and pressure fields. All cases (A to F) centerline temperature profiles are presented in Fig. 10. For the sea-level flight cases (A-C), we observe a strong difference of centerline pressure between the equilibrium (A) and nonequilbrium cases (B-C). This is expected as this set of cases rapidly reaches equilibrium compared to one flow-through time, causing a transfer of a 


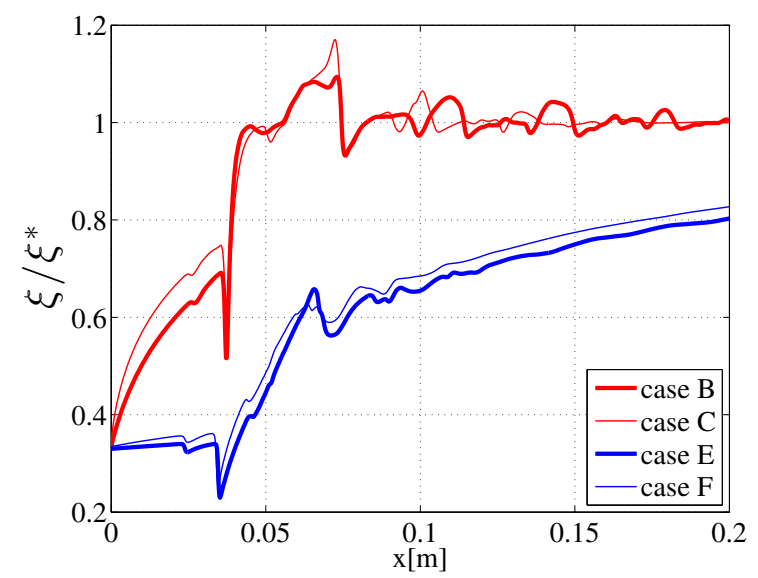

Figure 9. $\xi$ normalized by its local equilibrium value $\xi^{*}$.

substantial part of the internal energy into the vibrational modes, hence decreasing the translational energy, and the temperature. Additionally, Fig. 11 shows a similar effect on the pressure ratio $\mathrm{P} / \mathrm{P} 1$. This illustrates how relaxation drastically reduces the efficiency of the isolator, pumping energy into "sink modes", reducing the back pressure and the temperature rise we gradually recover from the shocks. The strong temperature drop can seriously affect the combustion rate and reduce the total thrust. In general, relaxation is going to become negligible at high altitude as the relaxation timescale becomes much larger than the residence time. When comparing cases D, E and F in both Figs. 10 and 11, it appears that the isolator length should be under $10 \mathrm{~cm}$ (i.e. 10 channel heights) to avoid excessive relaxation before entering the combustor, even for humid air. The tradeoff is therefore to scale the shock train size to slow the flow as much as possible (and recover a higher back pressure/temperature rise) while avoiding to lose energy to vibrational modes.
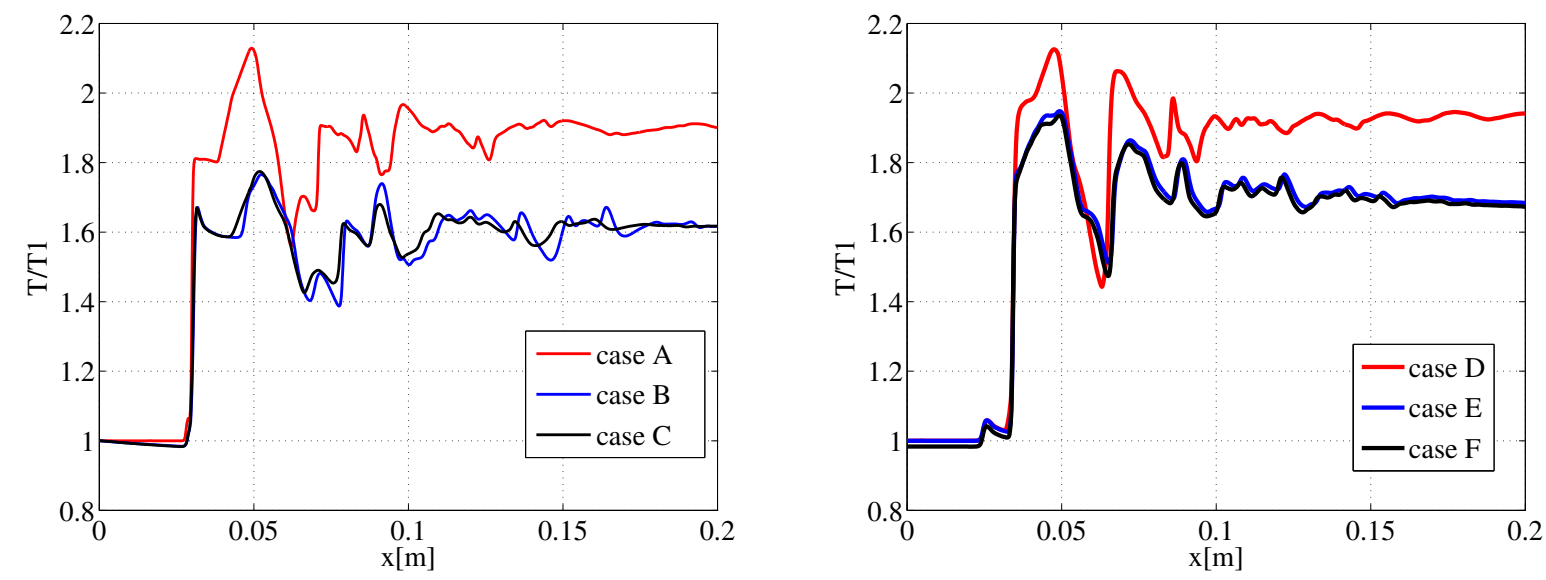

Figure 10. Centerline temperature ratio $\mathrm{T} / \mathrm{T} 1$.

Nonetheless, the strong variations observed between cases A, B and C warrant caution. Indeed they might partly be due to the uncertainty in the location of the reflection point of the oblique shock over the ramp, and the possible presence of a weak expansion fan at the turning angle. This expansion is actually observable in Fig. 4, indicating the overall geometry is not fully optimized, as we ideally wish the shock to entirely cancel out the expansion fan. The expansion wave is observed to reflect inside the channel while progressively fading, periodically inducing some local nonequilibrium to the flow as the regular bumps indicates in all case B centerline profiles in Fig. 9 for instance. Nevertheless cases D, E and F exhibits a very similar profile further inside the shock train, and we can therefore assume that the differences found by the end of the isolator between the centerline profiles accounts for nonequilibrium phenomena only. 

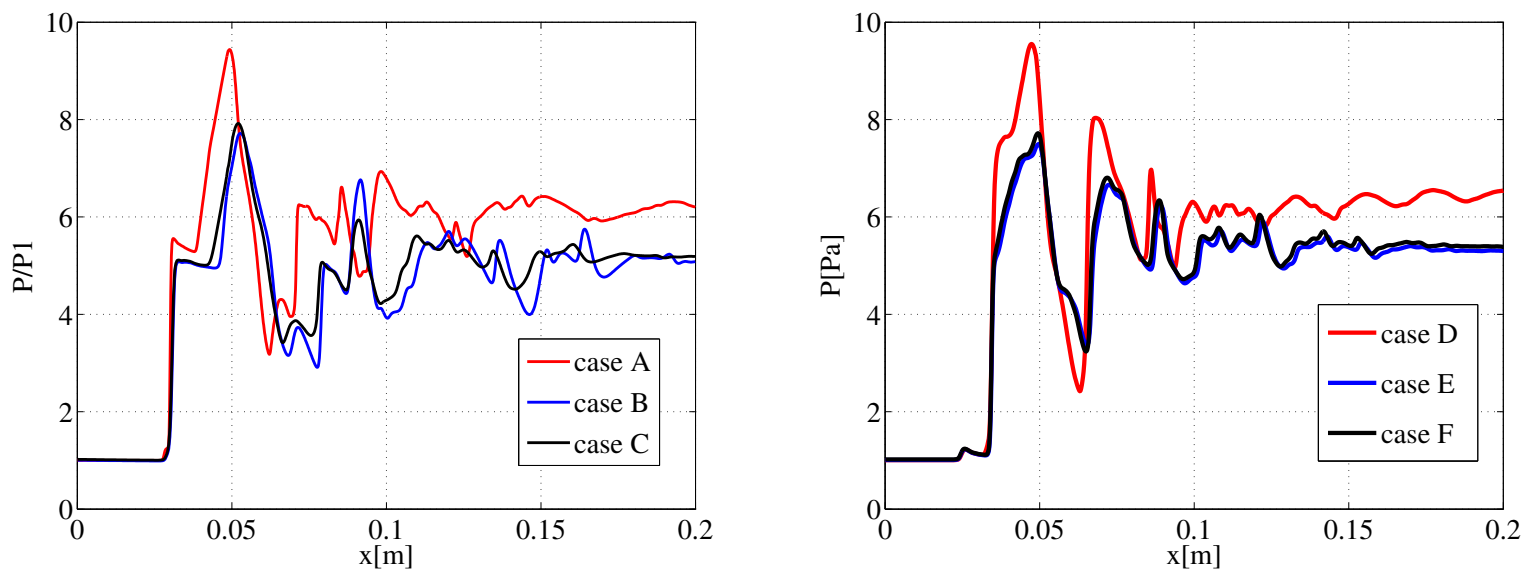

Figure 11. Centerline pressure ratio $\mathrm{P} / \mathrm{P} 1$.

\section{Conclusions}

The presence of nonequilibrium in isolators is studied using a simple linear relaxation model for the vibrational temperature. It is found that high altitudes, the low pressure conditions will contribute to significant non-equilibrium with considerable under-population of the vibrational modes. Chemical reactions that are sensitive to the vibrational modes will therefore be affected by this phenomenon. The presence of water vapor does decrease the level of nonequilibrium, but is not substantial. However, the chemical reactions related to fuel oxidation will release water vapor in much higher quantities that can effectively quench nonequilibrium. Consequently, this phenomenon is important for the fuel-air mixing as well as the initiation of chemical reactions, but is expected to subside in the post-combustion zone.

\section{Acknowledgements}

This work was financially supported through an AFRL grant FA9550-12-1-0460. The authors also gratefully acknowledge the generous allocation of computing time by the Texas Advanced Computing Center and NASA Pleiades supercomputer.

\section{References}

${ }^{1}$ Koo, H., Raman, V., and Varghese, Phillip, L., "Direct numerical simulation of supersonic combustion with thermal nonequilibrium," Proceedings of the Combustion Institute, 2014, pp. in press.

${ }^{2}$ Park, C., "Review of chemical-kinetic problems of future NASA missions. 1 - earth entries," Journal of Thermophysics and Heat Transfer, Vol. 7, No. 3, 1994, pp. 385-398.

${ }^{3}$ Candler, G. V. and MacCormack, R. W., "Computation of weakly ionized hypersonic flows in thermochemical nonequilibrium," Journal of Thermophysics, Vol. 5, No. 3, 1991, pp. 266-273.

${ }^{4}$ Knab, O., Fruhauf, H.-H., and Messerschmid, E. W., "Theory and validation of the physically consistent coupled vibration-chemistry-vibration model," Journal of Thermophysics and Heat Transfer, Vol. 9, No. 2, 1995, pp. $219-226$.

${ }^{5}$ Thivet, F., Perrin, M. Y., and Candel, S., "A unified nonequilibrium model for hypersonic flows," Physics of Fluids A, Vol. 3, No. 11, 1991, pp. 2799-2812.

${ }^{6}$ Millikan, R. C. and White, D. R., "Systematics of vibrational relaxation," Journal of Chemical Physics, Vol. 39, 1963, pp. 3209.

${ }^{7}$ Vincenti, W. G. and Kruger, C. H., Introduction to Physical Gas Dynamics, Krieger Pub. Co., 1975.

${ }^{8}$ Paull, A., Alesi, H., and Anderson, H., "The HyShot Flight Program and how it was developed," 41st AIAA Aerospace Sciences Meeting and Exhibit, No. AIAA-2002-4939, 2002.

${ }^{9}$ Koo, H., Donde, P., and Raman, V., "A Quadrature-based LES/Transported Probability Density Function Approach for Modeling Supersonic Combustion," Proceedings of the Combustion Institute, Vol. 33, 2011, pp. 2203-2210.

${ }^{10}$ Koo, H., Donde, P., and Raman, V., "LES-based Eulerian PDF approach for the simulation of scramjet combustors," Proceedings of the Combustion Institute, Vol. 34, No. 2, 2013, pp. 2093-2100.

${ }^{11}$ Donde, P., Koo, H., and Raman, V., "A multivariate quadrature based moment method for LES based modeling of supersonic combustion," Journal of Computational Physics, Vol. 231, No. 17, 2012, pp. 5805-5821.

${ }^{12}$ Jiang, G.-S. and Peng, D., "Weighted ENO Schemes for Hamilton-Jacobi Equations," SIAM Journal of Scientific Computing, Vol. 21, No. 6, 2000, pp. 2126-2143. 
${ }^{13}$ Koo, H. and Raman, V., "Large-Eddy Simulation of a Supersonic Inlet-Isolator," AIAA Journal, Vol. 50, No. 7, 2012, pp. $1596-1613$.

${ }^{14}$ Koo, H., Large-eddy simulations of scramjet engines, Ph.D. thesis, The University of Texas at Austin, 2011.

10 of 10 